\title{
"EL SOL NO SIEMPRE BRILLA PARA TODOS"1: ESTRATEGIAS DE INSERCIÓN DE LOS JÓVENES AFROCOLOMBIANOS A LA CIUDAD DE PEREIRA
}

\author{
Sandra Patricia Martínez Basallo*
}

\begin{abstract}
El acelerado proceso de urbanización de la población afrocolombiana puede explicarse a partir de los crecientes flujos migratorios desde la región Pacífica hacia las ciudades, entre las cuales, Pereira ha adquirido una relativa importancia en los últimos años como polo de atracción de esta población. No obstante, el fenómeno migratorio hacia esta ciudad así como los procesos de integración de los migrantes a la sociedad receptora, han recibido una escasa atención por parte de los analistas sociales. En tal sentido, este artículo espera contribuir al esclarecimiento de las estrategias de inserción urbana desplegadas por los migrantes afrocolombianos, haciendo especial énfasis en la experiencia migratoria de los jóvenes procedentes del departamento del Chocó, por ser éste uno de los grupos poblacionales mayormente afectado por dicho fenómeno. A la luz de este estudio de caso, se pretende demostrar que, en virtud de su pertenencia étnico-racial, origen regional y clase social, estos jóvenes enfrentan condiciones adversas en su proceso de integración a la vida urbana.
\end{abstract}

Palabras clave: proceso migratorio, estrategias de inserción urbana, jóvenes afrocolombianos, Pereira, Chocó.

\section{Introducción}

Diversos autores coinciden en señalar el acelerado proceso de concentración urbana de la población afrocolombiana que se viene presentando desde la década de los setenta del siglo anterior. Este proceso hace parte de una fuerte migración rural-urbana desde los municipios del Pacífico ${ }^{2}$ hacia ciudades

Profesora del Departamento de Ciencias Sociales, Universidad del Valle. Cali/Colombia.

1 Palabras de Pamela, en entrevista realizada en Pereira, el 26 de noviembre de 2011. Con el fin de proteger la identidad de los entrevistados, sus nombres reales han sido sustituidos por nombres ficticios.

2 La región Pacífica colombiana se extiende a lo largo de 960 kms de la costa occidental del país. De norte a sur está comprendida por la península del Darién, en límites con Panamá, hasta la frontera 
como Bogotá, Cali, Medellín, Tumaco y Buenaventura, que ha despertado el interés de diferentes analistas. No obstante, otras ciudades intermedias como Pereira, destino elegido por gran parte de los migrantes procedentes del Pacífico, en particular del departamento del Chocó, siguen siendo un campo por explorar.

El aumento de las corrientes migratorias hacia esta ciudad puede explicarse en virtud de la dialéctica entre unos factores de expulsión y atracción que impulsan a la población a moverse desde las regiones más deprimidas del país hacia aquellas que representan mejores oportunidades de vida. Entre los primeros, cabe señalar la precariedad en las condiciones de vida, la escasa oferta educativa, el restringido mercado laboral, los bajos niveles salariales y los desafueros del conflicto armado a los que se ven sometidos los habitantes de la región Pacífica. Entre los factores que han hecho de Pereira un polo de atracción poblacional, pueden contarse el repunte de la economía cañera y cafetera y el proceso de industrialización que tuvieron lugar en esta ciudad y su Área Metropolitana durante las décadas de los setenta y ochenta del siglo pasado, así como el mejoramiento en los índices de calidad de vida que se ha presentado en la ciudad en los últimos años. No obstante, la explicación del proceso migratorio de los afrocolombianos hacia esta ciudad no se agota en esta dialéctica expulsiónatracción. En este proceso, también han intervenido las redes construidas por los migrantes para afrontar su experiencia, las cuales han cumplido una importante función tanto en el mantenimiento del vínculo de éstos con sus regiones de origen, como en sus procesos de adaptación e integración a la ciudad destino.

Ahora bien, el incremento de los flujos migratorios de los afrocolombianos hacia la ciudad de Pereira así como los procesos de integración de esta población a la sociedad receptora han recibido una escasa atención por parte de los investigadores dedicados a estos temas. En tal sentido, este artículo espera contribuir al conocimiento de dicho fenómeno, a partir del análisis de las distintas estrategias de inserción que los afrocolombianos han desplegado en esta ciudad que, como veremos, se ven altamente condicionadas por su pertenencia étnicoracial, su origen regional y su clase social.

Para ello, hemos decidido centrar nuestro interés en el estudio de la migración interna de los afrocolombianos hacia Pereira, a la luz de la experiencia migratoria de los jóvenes chocoanos entre 18 y 25 años que se han trasladado en la última década hacia esta ciudad.

con Ecuador, y de este a oeste, desde el lado poniente de la cordillera occidental hasta el Océano Pacífico. Esta región comprende la totalidad del departamento del Chocó, el límite noroccidental de Antioquia, y las áreas occidentales de los departamentos del Valle del Cauca, Cauca y Nariño. El grueso de la población está constituido por descendientes de esclavos africanos, introducidos a la región durante el régimen colonial para la explotación minera. 
Para el logro de este propósito, entre septiembre de 2011 y febrero de 2012, realizamos trabajo de campo en la ciudad de Pereira, donde recorrimos algunos de los asentamientos en los que reside la población chocoana, en los cuales efectuamos entrevistas semi-estructuradas con los jóvenes seleccionados para el estudio, con base en unos tópicos previamente definidos, pero manteniendo el formato de preguntas abiertas con el fin de propiciar que los jóvenes expresaran en sus propias palabras sus perspectivas sobre los temas indagados. Asimismo, teniendo en cuenta que en la mayoría de los casos la decisión migratoria de los jóvenes se produce en el contexto de un proyecto migratorio familiar, en el proceso de realización de las entrevistas, procuramos apoyar sus testimonios en el de sus padres, tíos u otros familiares cercanos que se encontraban en el momento de la conversación.

Para la selección de los jóvenes a entrevistar, recurrimos al método de "bola de nieve". Así, inicialmente establecimos contacto con una profesora de la Universidad Tecnológica de Pereira - UTP - de origen afrodescendiente, quien nos presentó a algunas de sus primas residentes en la ciudad, específicamente en las comunas Del Café, Villa Santana y Boston (barrios Parque Industrial, Villa Santana y La Unidad, respectivamente). A su vez, una de ellas nos puso en contacto con otros jóvenes que fueron entrevistados en la Ciudadela Tokio, perteneciente a la Comuna Villa Santana. Igualmente, esta profesora nos facilitó el acercamiento a algunos de los estudiantes chocoanos que hacen parte del Programa de Etnoeducación y Desarrollo Comunitario de la UTP, quienes fueron entrevistados en el campus de esta universidad.

Por tratarse de una investigación de orden cualitativo, el tamaño de la muestra no se fijó con anticipación, sino que éste se fue configurando a través de las diferentes etapas del proceso de recolección de datos, es decir, el acopio de la información primaria se dio por terminado una vez que se llegó a un punto de saturación de los datos, reflejado en el surgimiento de patrones recurrentes que no agregaban nueva información a la ya acopiada. Fue así como realizamos 12 entrevistas semi-estructuradas a seis mujeres e igual número de varones afrocolombianos entre 18 y 25 años, procedentes del departamento del Chocó. Su tiempo de residencia en Pereira oscila entre los dos y los 10 años. En cuanto a su grado de escolaridad, tres de ellos no han culminado sus estudios de bachillerato, el mismo número son bachilleres, mientras que seis están cursando estudios universitarios. Siete de ellos están estudiando, cuatro se encuentran desempleados y una laborando como empleada del servicio doméstico. Cuando las circunstancias lo permitieron, recabamos información con los familiares de los jóvenes migrantes. Adicionalmente, realizamos ocho entrevistas con cuatro mujeres y el mismo número de varones afrocolombianos que si bien no se ajustaban al grupo etáreo inicialmente fijado, aportaron información valiosa 
en el marco de la investigación. El tiempo de residencia de estos migrantes en Pereira oscila entre los cuatro y los 14 años.

De otra parte, se sostuvieron entrevistas o conversaciones informales con dos profesores universitarios de la UTP y uno de la Fundación Universitaria del Área Andina, cuyo conocimiento de este tema se consideró relevante para el estudio. Es importante señalar cómo el conocimiento previo que teníamos de los lugares de origen de esta población, nos facilitó entablar conversaciones de carácter informal con un par de migrantes chocoanas residentes en estos barrios, gracias a las cuales pudimos construir los relatos de vida relativos a su experiencia migratoria. Es preciso mencionar también las entrevistas efectuadas al representante de la organización estudiantil Afro UTP y a dos funcionarios de la Alcaldía de Pereira, quienes brindaron información sobre los estudiantes chocoanos matriculados en la UTP, las políticas de vivienda de interés social, así como sobre los programas para la generación de empleo adelantados por esta entidad, respectivamente.

Los tópicos abordados en las entrevistas con los jóvenes giraron en torno a cuatro grandes aspectos. El primero de ellos se dirigió a establecer un perfil general del migrante, a partir de categorías como género, edad, adscripción étnica, lugar de nacimiento, lugar de procedencia y nivel educativo. El segundo aspecto indagado contempló la composición familiar de los migrantes tanto en el lugar de salida como en la ciudad, así como la ocupación de sus allegados. Un tercer componente de la entrevista giró en torno a la situación del migrante antes de su traslado hacia Pereira: ocupación en el lugar de origen; motivaciones que lo impulsaron a abandonar su sitio de origen; migración hacia otros lugares, tiempo de permanencia en ellos y ocupaciones desempeñadas; tipo de ayudas recibidas por parte de familiares, amigos o vecinos para trasladarse hacia la ciudad; información previa sobre el lugar de destino y horizonte temporal de la migración. El cuarto y último aspecto abordado en las entrevistas fue el proceso de inserción en la ciudad, para lo cual se inquirió por el año de llegada a la ciudad; la ocupación desempeñada al momento de la entrevista; el barrio de residencia; el papel de las redes étnicas en los procesos de integración a la vida urbana; las condiciones de llegada a la ciudad en cuanto a vivienda, empleo y opciones educativas; el grado de satisfacción de la expectativas que se abrigaban respecto al lugar de destino; la migración de otros familiares, amigos o conocidos hacia la ciudad, así como las expectativas de retorno a los lugares de origen. El tiempo de duración de cada entrevista osciló entre los 45 minutos y una hora y media. En el caso de los informantes clave, quienes además prestaron su colaboración para establecer contacto con nuevas personas a entrevistar, fue posible sostener varias conversaciones a través de charlas de carácter más informal que el evento de la entrevista. 
Antes de pasar a la presentación del estudio de caso, dedicaremos unas cuantas páginas a las contribuciones que distintos autores han hecho a la comprensión del proceso migratorio de la población afrocolombiana hacia distintas ciudades del país, particularmente en lo que tiene que ver con las dinámicas espaciales, residenciales y laborales asociadas a este proceso. Posteriormente, veremos cuáles han sido las estrategias de inserción de los jóvenes chocoanos a la ciudad de Pereira, en términos de la ocupación espacial y de los mecanismos de acceso a vivienda y empleo. Por último, nos centraremos en las expectativas de los jóvenes respecto a su proyecto de vida, las cuales se encuentran profundamente mediadas por su situación de clase. Los testimonios de estos jóvenes se hacen eco de algunos de los principales conceptos de la teoría bourdeana, por lo que recurriremos a este autor para iluminar el análisis.

\section{La migración de los afrocolombianos a las ciudades: procesos de ocupación espacial y residencial e inserción laboral}

Uno de los estudios pioneros en el abordaje de la migración de los afrocolombianos hacia las ciudades es la tesis doctoral de Peter Wade que, traducida al español con el nombre de Gente Negra, nación mestiza: dinámicas de las identidades raciales en Colombia, se propone comprender la naturaleza del orden racial colombiano. Según el autor, la estructura de este orden se asemeja a un triángulo, en cuyo ápice se localiza lo blanco, asociado con el poder, la civilización y la riqueza, mientras que en sus dos vértices inferiores se sitúan las poblaciones afrocolombianas e indígenas, las cuales son visualizadas como primitivas, ignorantes y rústicas ${ }^{3}$. A esta estructura se superpone un orden de desigualdades regionales, donde las zonas predominantemente blancas y mestizas son definidas como progresistas, mientras que aquellas en las que se concentra la población afrocolombiana, son calificadas de atrasadas y subdesarrolladas. Para Wade, estos órdenes estructurales afectan los procesos de inserción de los afrocolombianos en la ciudad, quienes además de enfrentarse a la discriminación racial, deben encarar la construcción de determinados estereotipos sobre su origen regional. A la luz del estudio de caso de la migración de chocoanos a Medellín, el autor concluye que estos migrantes se insertan de manera subalterna a un contexto definido como superior en las jerarquías de raza, poder y cultura ${ }^{4}$.

Siguiendo la línea de análisis planteada por Wade, Barbary y Urrea señalan que las relaciones sociales en Colombia se hallan inscritas en un orden racial jerarquizado que, como tal, es productor de desigualdades sociales, susceptibles de constatación en distintos ámbitos como el residencial, el laboral

WADE, Peter. Gente Negra, nación mestiza: dinámicas de las identidades raciales en Colombia, p. 52.

4 Ibidem, p. 226. 
y el espacial. Estos mecanismos de discriminación se conjugan con otros factores de diferenciación tales como el origen migratorio, la clase social, la edad y el género ${ }^{5}$, cuya intensidad se expresa en dinámicas particulares de segregación y segmentación en términos de ingresos y de acceso al mercado laboral.

Con relación a la inserción de los afrocolombianos al mercado laboral urbano, las investigaciones efectuadas en distintas ciudades como Medellín, Cali y Bogotá ${ }^{6}$ coinciden en señalar que esta población, por lo menos inicialmente, sólo puede competir en los niveles más bajos de la escala ocupacional, viendo reducidas sus posibilidades de movilidad ascendente. Como tendencia general, estos estudios identifican un patrón ocupacional por sexos, siendo predominante el enganche de las mujeres al servicio doméstico y a otros oficios no calificados en el sector servicios, mientras que los hombres se emplean como obreros manufactureros y de la construcción, artesanos, pintores, conductores, zapateros, entre otros oficios. La vinculación de esta población al sector informal de la economía es común a los dos sexos. Además de estas ocupaciones, es frecuente el empleo de los afrocolombianos en la fuerza policial, la cual es visualizada como un medio relativamente fácil de ascenso social, por las posibilidades que ésta ofrece de obtener un ingreso fijo y un estatus de autoridad, sin exigir altos grado de escolaridad.

En cualquier caso, las redes étnicas de parientes, amigos, vecinos o paisanos juegan un papel central, no solamente en la búsqueda de estrategias de sobrevivencia, sino también como puntos de apoyo a través del alojamiento temporal de los migrantes recién llegados, el préstamo de dinero y ropa, el respaldo moral y el cuidado de los hijos. Con el tiempo, la función de las redes como fuentes de información y contactos para la consecución de trabajo tiende a ser sustituida por mecanismos formales como el envío de hojas de vida a las empresas o agencias de empleo, o la presentación personal. Otros migrantes por su parte, se lanzan al llamado "rebusque" mediante el establecimiento de ventas callejeras?.

De otra parte, las investigaciones en esta materia han demostrado que las prácticas de discriminación racial determinan condiciones desfavorables en el acceso a oportunidades laborales y de ingresos para la población afrocolombiana. Los estudios de Wade y Posso muestran cómo las ciudades de Medellín y Cali, respectivamente, presentan mercados de trabajo caracterizados por patrones de segregación, donde el color de la piel prescribe la inserción de los afrocolombianos en las posiciones más bajas de la estructura ocupacional. En el caso particular de

BARBARY, Olivier; URREA, Fernando. Gente negra en Colombia. Dinámicas sociopolíticas en Cali y el Pacífico, p. 32.

6 WADE, op. cit.; MOSQUERA, Claudia. Estrategias de inserción de la población negra en Santafe de Bogotá. Acá antes no se veían negros; POSSO, Jeanny. La inserción laboral de las mujeres inmigrantes negras en el servicio doméstico de la ciudad de Cali.

MOSQUERA, op. cit.; POSSO, op. cit. 
la vinculación de las mujeres al servicio doméstico, estas investigaciones develan la experiencia de discriminación que, por su condición de raza, género y clase, sufren estas mujeres en su proceso de inserción al mercado de trabajo urbano.

En la misma dirección, Urrea, Viáfara y Correa analizan las desigualdades sociodemográficas y socioeconómicas que enfrenta la población afrocolombiana en relación con la blanca y mestiza, así como el papel que estas iniquidades desempeñan en la inserción de aquella población al mercado laboral. Los autores demuestran que la desigualdad de oportunidades encarada por los afrodescendientes se traduce en menores dotaciones de capital humano, en términos de acceso a los servicios de educación, salud y saneamiento básico, afectando negativamente su inserción al mundo del trabajo. Pero además de estas desigualdades, los autores advierten que las posibilidades de movilidad social ascendente para los afrocolombianos se ven restringidas por el trato discriminatorio del que éstos son objeto en el medio laboral. Aún en aquellos casos en que no se registran diferencias significativas en el nivel educativo y de cualificación entre los afrocolombianos y los blanco-mestizos, los primeros encuentran condiciones desventajosas en cuanto a sus posiciones ocupacionales, niveles salariales y posibilidades de promoción en el empleo ${ }^{8}$.

En términos de la distribución espacial y residencial de los afrocolombianos en las ciudades, diferentes estudios señalan la coexistencia de un patrón que tiende a la concentración de esta población en determinados barrios, con otro que se dirige hacia su dispersión en toda la ciudad. En la elección del lugar de residencia, intervienen diversos factores como el grado de consolidación económica y de estabilidad laboral, el tiempo de residencia en la ciudad, el peso relativo de las redes étnicas y el ciclo familiar. Así, la formación de núcleos afrocolombianos es más frecuente entre los migrantes recientes y de más bajos ingresos, mientras que la tendencia a la dispersión se encuentra asociada a niveles económicos más altos y a un mayor tiempo de residencia en la ciudad. Este proceso tiene como correlato ciertas lógicas de distribución espacial urbana, a las cuales subyace una doble dinámica: aquélla a partir de la cual se crean núcleos de asentamiento, promovidos en gran parte por las redes étnicas y, las lógicas de dispersión e integración a la vida en la ciudad, donde los vínculos con la red tienden a debilitarse ${ }^{9}$.

De manera consistente con las lógicas de distribución espacial en las ciudades, las estrategias de los migrantes afrocolombianos para resolver el acceso a la vivienda son diversas. En la ciudad de Medellín, Wade identificó la

${ }_{8}$ URREA, Fernando; VIÁFARA, Carloas; CORREA, Juan. Desigualdades sociodemográficas y socioeconómicas, mercado laboral y discriminación étnico-racial en Colombia: análisis estadístico como sustento de Acciones Afirmativas a favor de la población afrocolombiana, p. 154-155.

9 WADE, op. cit.; MOSQUERA, op. cit.; AGIER, Michel et alii. Espacios regionales, movilidad y urbanización, dinámicas culturales e identidades de las poblaciones afrocolombianas del Pacífico sur y Cali. 
invasión de lotes baldíos, el alquiler de habitaciones en inquilinatos, la compra de lotes a urbanizadores pirata y el traslado a otros barrios con mayores grados de consolidación y acceso a servicios públicos, en el caso de aquellos migrantes que cuentan con un mejor nivel económico. En Bogotá, Mosquera encontró la coexistencia de familias numerosas en viviendas compartidas, dadas las ventajas económicas representadas en este tipo de arreglos, donde los gastos de sostenimiento se reparten entre varios proveedores.

Para el caso de Cali, el equipo conformado por el Centro de Investigaciones y Documentación Socio-económica de la Universidad del Valle - Cidse - y por el Instituto de Investigación para el Desarrollo - IRD - comprobó que la segmentación espacial del espacio urbano en esta ciudad coincide con un alto grado de segregación racial, específicamente de la población caracterizada como afrocolombiana. En consecuencia, estos hogares tienden a concentrarse en las zonas más deprimidas de Cali, donde su acceso a bienes y servicios es precario, y sus índices de Necesidades Básicas Insatisfechas - NBI -, altos ${ }^{10}$. Wade encontró patrones similares en Medellín, en donde a partir de una investigación etnográfica, el autor concluyó que si bien la segregación espacial de los chocoanos en la ciudad puede atribuirse en gran parte al nivel de ingresos de estos migrantes, también es adjudicable a la discriminación racial, aunque ésta no sea ejercida de manera abierta y sistemática, sino sutilmente, en consonancia con la dinámica del orden racial colombiano ${ }^{11}$.

En síntesis, estas investigaciones coinciden en señalar que el proceso de inserción de los afrocolombianos a los contextos urbanos se ha producido en condiciones de subordinación, toda vez que dichas poblaciones se hallan inmersas en un círculo vicioso, donde las desigualdades impuestas por su origen regional y étnico redundan en un acceso diferencial al capital económico, educativo y cultural, el cual a su vez se traduce en menores oportunidades de competir en el mercado de trabajo, y por ende, de acceder a una mejor calidad de vida en la ciudad. La situación descrita por estos autores no es distinta a la encontrada entre los chocoanos residentes en Pereira que, como veremos, enfrentan limitadas posibilidades de inserción al mundo laboral y no menos precarias condiciones de acceso a servicios como la vivienda.

\section{El difícil proceso de inserción laboral de los jóvenes chocoanos a la ciudad}

En relación con las dinámicas de inserción de los jóvenes afrocolombianos al mercado de trabajo en Pereira, los hallazgos de este trabajo coinciden en gran parte con los obtenidos por los investigadores anteriormente referidos.

\footnotetext{
${ }^{10}$ BARBARY, Olivier. Observar los hogares afrocolombianos en Cali, problemas teóricos y metodológicos ilustrados, p. 3; BARBARY, URREA, op. cit., p. 107.

${ }^{11}$ WADE, op. cit., p. 262.
} 
Es así como el servicio doméstico se ha constituido en uno de los principales mecanismos de inserción al mundo laboral urbano para las jóvenes chocoanas. Cabe señalar también su vinculación a servicios de aseo en empresas privadas, labores de cocina en restaurantes o como vendedoras en almacenes. Los hombres por su parte, han encontrado en el sector de la construcción y en la recolección del café, sus principales actividades ocupacionales. Las ventas ambulantes constituyen también una fuente de ingresos para unos y otras.

En los últimos dos años, el Plan de Choque para la generación de empleo, implementado por la Alcaldía de Pereira en coordinación con el Departamento Administrativo para la Prosperidad Social ${ }^{12}$, se ha constituido en una importante fuente de trabajo para los migrantes chocoanos. Destinado a familias de escasos recursos, con un periodo de desempleo superior a un año o en situación de desplazamiento, el Plan de Choque ha generado cerca de 2000 empleos directos en la construcción de obras viales menores, mantenimiento de laderas y quebradas y, montaje y mejoramiento de casetas comunales ${ }^{13}$. Llama la atención la vinculación de las mujeres a actividades que, como la construcción, han sido tradicionalmente calificadas como masculinas.

Las estrategias de vinculación laboral descansan, por lo menos inicialmente, en la red de familiares, amigos o conocidos, quienes asisten al joven migrante en su proceso de inserción en las mismas actividades ocupacionales que éstos desempeñan. Otros chocoanos por su parte, han optado por lanzarse de manera independiente a la búsqueda de empleo a través del envío de hojas de vida, estrategia que difícilmente arroja los resultados esperados, dada la discriminación racial que opera en los procesos de selección de personal. Asimismo, algunos de los entrevistados aluden el recurso a las redes clientelistas como una de las estrategias utilizadas en la consecución de trabajo.

En cuanto a la situación laboral, las jóvenes chocoanas que han tenido la experiencia de trabajar como empleadas del servicio doméstico coinciden en señalar las desventajosas condiciones de contratación imperantes en este sector, las cuales se ven representadas en los bajos niveles de remuneración salarial, la ausencia de garantías laborales tales como el pago de prestaciones sociales, el reconocimiento de periodos vacacionales y la cancelación de una liquidación al momento de la terminación del vínculo laboral. Con relación a los salarios, algunas de las entrevistadas subrayan el contraste existente entre las ciudades en términos de las remuneraciones devengadas. Así por ejemplo, el nivel salarial en Bogotá es superior al de Pereira y éste a su vez es mejor si se compara con el de ciudades

$\overline{12}$ Entidad a cargo de las políticas de asistencia, atención y reparación dirigidas a las víctimas de la violencia y otros grupos vulnerables.

${ }^{13}$ Información suministrada por un funcionario de la Oficina de Infraestructura de la Alcaldía de Pereira. Pereira, 03.02.2012. 
como Quibdó (capital del departamento del Chocó), donde una empleada del servicio doméstico tan sólo recibe una tercera parte de lo que devenga en Bogotá. Deisy, una joven de 25 años que tuvo que desplazarse a raíz del recrudecimiento del conflicto armado en su lugar de origen, narra de la siguiente manera su experiencia como empleada doméstica en esta última ciudad:

\section{E: ¿Acá cuánto pagan?}

D: Aquí cuando mucho hay un trabajo de trescientos mil pesos, entonces a usted la quincena le sale en ciento cincuenta y allá en Bogotá le pagan a usted seiscientos mensual y la quincena le sale en trescientos, entonces mire usted la diferencia que le pagan más allá que acá. Acá no pagan nada. Un día son cuarenta que pagan allá, entonces uno tiene que mirar la diferencia.

E: Y iaquí cuánto pagan por el día?

D: El día cuando mucho $\$ 12.000, \$ 13.000$.

E: Y ipagan servicio de salud?

D: No. Aquí uno no tiene (...) afuera en las afueras sí, digamos que uno sale de aquí para Bogotá si le dicen a uno: 'quiero que tengas un seguro porque en una caída o en cualquier accidente...'

E: ¿Y te han tratado bien?

D: Sí, no tengo nada que decir de ellas. Pues algunas no le pagan pues la plata a uno, otras se van.

E: ¿No les pagan?

D: No, algunas me han quedado debiendo. El año pasado trabajé como nueve meses en una casa y no me pagaron la liquidación y parte de la plata no me la pagaron, como digo, eso yo nunca demandé, ni digo nada, solamente arriba hay un Dios y Él lo ve todo. ${ }^{14}$

A estas condiciones, se suma el trato discriminatorio que por su condición étnico-racial, han tenido que enfrentar muchas de ellas. Sin embargo, tal situación no es exclusiva del ámbito del servicio doméstico, sino que se extiende a los diferentes espacios de trabajo en los que se emplean los chocoanos. Así por ejemplo, Camilo, un joven de 24 años que migró a la ciudad hace seis años con el propósito de realizar sus estudios superiores, decidió renunciar a su empleo en una empresa de marketing, tras encontrarse con las actitudes racistas de los habitantes de los barrios de élite a donde debía dirigirse en búsqueda de clientes:

(...) Y también estuve trabajando en una empresa de marketing que como que le hacía promociones a otras empresas, entonces ellos diseñaban los bonos de promociones y uno vendía eso bonos [...] y entonces eso allí uno se ganaba por comisión, y otro trabajo que yo decía que yo allí no me iba a sacrificar porque era demasiado y uno ofrece mucho el cuerpo y se desgasta uno demasiado, porque tocaba prácticamente desde las ocho de

\footnotetext{
${ }^{14}$ Entrevista realizada a Deisy. Pereira, 27.11.2011. Un real equivale a 890 pesos colombianos.
} 
la mañana hasta las cinco de la tarde, uno estar recorriendo esta ciudad, uno ofreciendo toda esta clase de cosas, y entonces pedían un tipo de ropa que uno tenía que ir vestido, zapatos serios, pantalón serio, camisa larga, corbata, entonces yo para eso no estaba, y además, veían a uno así, todo pintoso, y además decían, yo me imagino, estoy hablando para el otro, 'vea ese negro que viene allá, encorbatado, además en este barrio de estrato seis, éste no viene a hacer cosas buenas por acá'. ${ }^{15}$

Pero la condición étnico-racial de los jóvenes no sólo incide en las relaciones que éstos construyen en los espacios de trabajo en los que se desenvuelven, sino que muchas veces se constituye en una barrera que dificulta, cuando no obstaculiza, el acceso mismo al empleo, tal y como lo refiere Paula, una joven chocoana de 25 años que arribó a la ciudad durante el último lustro:

(...) por acá como que no se ha acabado de vencer eso, el racismo, todavía está vigente, o sea, uno como que le nota a las personas, porque yo muchas veces he ido a una entrevista de trabajo y: 'iah! es una negra', ya como que se les cae el ánimo, a veces. Como hay gente que no le importa, 'iah! que pase, lo que sea, no importa el color que sea', y se le nota la amabilidad, pero hay otros que son un poco racistas. ${ }^{16}$

\section{"Esta ciudad es dura"17: procesos de ocupación urbana}

Con el abandono de sus lugares de origen, los chocoanos han emprendido también el difícil proceso de recomenzar sus vidas en las ciudades, entrando a formar parte del enjambre de migrantes rurales que luchan por un espacio en el paisaje urbano. Es así como la experiencia migratoria de la gente chocoana no ha sido ajena a los procesos que han configurado la expansión de la ciudad de Pereira desde la segunda mitad del siglo XX: invasiones o tomas de tierras, autoconstrucción y urbanizaciones piratas que, sumados a las acciones urbanizadoras emprendidas por las instituciones oficiales y la empresa privada, han dado origen a las 19 comunas y 421 barrios que actualmente conforman la ciudad. Sería totalmente ajeno al presente contexto intentar reconstruir este proceso en su conjunto, por lo que nos limitaremos a describir los principales factores que han dado lugar al surgimiento de los barrios que han hecho parte del periplo intraurbano seguido por nuestros entrevistados.

Los testimonios de los jóvenes migrantes refieren itinerarios residenciales marcados por distintas estaciones en asentamientos barriales diseminados a lo largo y ancho de la ciudad, con orígenes igualmente disímiles: desde aquellos

\footnotetext{
${ }^{15}$ Entrevista realizada a Camilo. Pereira, 01.02.2012. Pintoso es una expresión coloquial utilizada para designar a una persona que tiene buena presencia, por apariencia corporal o por vestimenta.

${ }^{16}$ Entrevista realizada a Paula. Pereira, 11.12.2011.

17 Palabras de Horacio, padre de uno de los jóvenes migrantes, en entrevista realizada en Pereira el 02.02.2012.
} 
conformados por las oleadas de migrantes expulsados del campo en el periodo de "La Violencia"18, hasta los ocupados en las últimas décadas por las familias desplazadas a causa del conflicto armado de nuestros días, pasando por aquellos otros que deben su nacimiento al proselitismo político, al inescrupuloso afán de lucro de los urbanizadores pirata o a los programas estatales que, de manera muchas veces tardía, han intentado "organizar" el espacio urbano.

De acuerdo con los factores que han dado lugar a la creación de los asentamientos por los que han trasegado los migrantes, tendríamos tres grandes categorías de barrios. En primer lugar, estarían aquellos que deben su nacimiento a los procesos de tomas de tierras, generalmente en zonas de alto riesgo, que han sido emprendidos por diferentes actores dependiendo del momento histórico en el que se han producido. Dentro de este grupo, se encuentra el sector de Cuba, en donde fueron reubicadas las familias campesinas que, huyendo de la violencia bipartidista de mediados del siglo pasado, se asentaron a orillas del Río Otún ${ }^{19}$. También harían parte de este grupo Nacederos y El Plumón Intermedio, surgidos a partir de la ocupación de los terrenos públicos del Ferrocarril de Occidente, desmontado por orden del gobierno nacional en el año de $1972^{20}$. Del mismo modo, barrios como el Plumón Alto, La Platanera, La Laguna, El Dorado, San Nicolás, Bosques de Combia y Bosques del Otún, los cuales empiezan a crearse a finales de la década de los noventa a partir de las invasiones de tierras protagonizadas por el creciente número de familias desplazadas que han arribado a la ciudad ${ }^{21}$.

En una segunda categoría, pueden agruparse los barrios que tienen su origen en la compra de tierras a particulares quienes, sin contar con una clara tradición de dominio sobre estos predios, los vendieron a familias de escasos recursos que hoy enfrentan dificultades a la hora de acreditar la propiedad legítima sobre los mismos. Los barrios El Plumón Bajo y Caracol La Curva hacen parte de esta categoría. En el caso del primero, los predios pertenecientes a este sector fueron vendidos a sus moradores por un particular, tras cuyo fallecimiento, sobrevinieron una serie de litigios en torno a la propiedad de los mismos, lo cual retardó el otorgamiento de las escrituras a sus compradores. Por su parte, el sector de Caracol La Curva pertenecía a los propietarios de las fincas productoras de café y plátano localizadas en esta zona, quienes les donaron lotes a sus trabajadores para que construyeran sus viviendas a la orilla del camino. Éstos últimos, que

\footnotetext{
18 "La Violencia" es la denominación utilizada para referirse al periodo de la historia colombiana comprendido entre 1947 y 1965, en el que la población rural se vio enfrentada en un conflicto violento a nombre de los dos partidos políticos tradicionales del país: el liberal y el conservador.

19 TOBÓN, Alfredo. Barrio Cuba, una ciudad dentro de otra.

20 TROYA, Marcela. Entre el recuerdo y el presente del Aeropuerto Internacional Matecaña; HENAO, Marisol. Una aproximación al desarrollo de habilidades comunicativas en la comunidad desplazada "del Plumón Alto" a través de la comunicación asertiva, p. 48.

${ }^{21}$ DE LOS MILAGROS, Camilo. Pereira: etimología de las invasiones.
} 
nunca formalizaron su posesión sobre dichos predios a través de la expedición de los respectivos títulos de propiedad, empezaron a venderlos a otros pobladores, a quienes se les han presentado una serie de inconvenientes para legalizar sus posesiones ${ }^{22}$. Cabe mencionar el acelerado crecimiento que ha tenido este último sector en años recientes, dada la llegada progresiva de familias en situación de desplazamiento, muchas de ellas procedentes del departamento del Chocó.

En tercer lugar, se encuentran aquellos barrios que surgen a partir del proselitismo de algunos políticos que han adquirido tierras en zonas periféricas de la ciudad, para posteriormente lotearlas y venderlas a inmigrantes de otras regiones del país o a familias de bajos recursos del mismo casco urbano de Pereira. A este grupo pertenecen sectores como Villa Santana, edificado a partir de los procesos de autoconstrucción emprendidos por sus habitantes a comienzos de los ochenta ${ }^{23}$, hasta llegar a constituirse en la comuna que hoy por hoy aglutina 19 barrios de estratos 1 y 2 .

La respuesta de la Administración Municipal a estos procesos de ocupación se ha traducido en el diseño e implementación de distintos programas de vivienda de interés social enmarcados en la política "El Camino hacia un país de propietarios" instaurada por el gobierno del Ex presidente Álvaro Uribe en el año 2000. El resultado de estos programas no ha sido el esperado, ya que si bien éstos le han permitido a cientos de familias acceder a una vivienda, la calidad de las casas entregadas, así como de los entornos habitacionales, no ha sido la mejor, toda vez que éstos adolecen de la infraestructura necesaria para la prestación de los servicios básicos de salud, educación y transporte público. En cierto modo, los grandes beneficiarios de estos planes de vivienda han sido los gremios de la construcción, quienes se han lucrado de las plusvalías generadas por la renta del suelo, a costa de los municipios, en los cuales ha recaído gran parte de la carga presupuestal, y de los mismos usuarios, quienes han tenido que asumir la terminación de sus viviendas, así como el sobrecosto que el cambio de estrato socioeconómico ha implicado en el pago de las facturas de servicios públicos. Lo anterior puede explicarse en virtud de la mirada que se tiene de los destinatarios de estas políticas:

(...) casi siempre estas soluciones de vivienda se están consolidando en la periferia de las ciudades, la segregación socio espacial genera en el territorio procesos de exclusión, cuando no se tiene una visión de integración a la ciudad y no se mira a las comunidades beneficiarias de

22 HENAO, op. cit., p. 49; CALDERÓN, Juan. Propuesta de constitución legal y administrativa para un grupo de mujeres en condición de vulnerabilidad económica, habitantes de los Barrios Caracol La Curva y El Bosque, aledaños al "Salado de Consotá", Pereira, p. 17.

${ }^{23}$ LÓPEZ, Santiago; SOTO, Stefany. Gestión ambiental urbana en programas de vivienda popular; estudio de caso "Ciudadela Tokio", Pereira, Risaralda, p. 17. 
la Vivienda de Interés Social como ciudadanos con derechos, sino como ciudadanos de categorías inferiores. ${ }^{24}$

Un claro ejemplo de esta política lo constituye la ciudadela Tokio, localizada en el extremo suroriental de Pereira, en donde han sido reubicados 925 hogares de escasos recursos, de los cuales 772 provienen de asentamientos ubicados en zonas de alto riesgo, entre ellos Bosques del Otún y Bosques de Combia, y 153 corresponden a familias en situación de desplazamiento, la mayoría de ellas asentadas en el Plumón Alto ${ }^{25}$. El proyecto contempló la entrega de un lote de $54 \mathrm{~m}^{2}$ (28 $\mathrm{m}^{2}$ construidos), dotado de los servicios públicos básicos, bajo la consideración de que sus beneficiarios culminarían la construcción y adecuación de sus viviendas. Asimismo, la urbanización cuenta con espacios públicos y un equipamiento colectivo constituido por una escuela, un parque, un centro deportivo y una cancha. No obstante, su localización en una zona periférica de la ciudad, así como la falta de pavimentación de sus calles han dificultado el acceso de sus habitantes al transporte público, al tiempo que han favorecido la emergencia de problemas de seguridad en el sector.

Además de la ciudadela Tokio, actualmente la Alcaldía de Pereira adelanta la construcción del proyecto habitacional El Remanso, también localizado al suroriente de la ciudad. Hasta la fecha, se han reubicado1221 familias procedentes de los barrios Avenida del Río, La Cascada, La Platanera, Caracol La Curva, La Laguna y El Plumón Bajo, los dos últimos afectados por la ocurrencia de incendios en 2008 y 2010, respectivamente. Cerca del 20\% de la población reubicada es desplazada, mientras que el porcentaje restante corresponde a habitantes de zonas de alto riesgo, así como a las familias damnificadas por dichos siniestros ${ }^{26}$. Por el momento, 1336 hogares se encuentran a la espera de ser reubicados, una vez culmine la edificación del proyecto.

De manera paralela a los programas de la Alcaldía, Prosperidad Social ha venido adelantando programas de dotación de vivienda a las familias en situación de desplazamiento, ya sea a través de la reubicación de estos hogares en sectores como Las Brisas y Málaga, o bien mediante la entrega de subsidios a las familias, a fin de que éstas adquieran directamente sus viviendas.

El profesor Palacios, procedente de Buenaventura y quien llegó a la ciudad en la década de los ochenta con el propósito de realizar sus estudios universitarios, ofrece una lectura crítica de los propósitos que han animado las políticas de reubicación implementadas por la Alcaldía de Pereira:

\footnotetext{
${ }^{24}$ Ibidem, p. 32.

${ }^{25}$ RIVERA, Alberto. La reubicación es un hecho.

${ }^{26}$ Información suministrada por un funcionario de la Oficina de Gestión Inmobiliaria de la Alcaldía de Pereira, entrevistado en Pereira el 3 de febrero de 2012.
} 
(...) entonces van apareciendo los barrios étnicos, entonces uno de esos barrios étnicos fundamentalmente es el barrio que quedaba cerca al aeropuerto, El Plumón, ahí va a aparecer el primer barrio étnico que luego es trasladado a lo que hoy se conoce como Villa Santana, se traslada allá. Luego aparece La Laguna, es una historia reciente, La Laguna termina incendiada, uno no sabe si el incendio es motivado por un ataque racista, pero termina incendiado. Otro barrio étnico es La Platanera que queda al lado del... La suerte de estos barrios es que sistemáticamente son trasladados... iojo!, una característica bien importante de estos lugares marcados por la gente negra desplazada es que no están ubicados en los márgenes, sino que están ubicados en la parte central, en la parte céntrica de la ciudad como tal, entonces la manera como se levantan las casas, la condición marginal se ve, la condición de aislamiento se ve, entonces hay presiones urbanísticas que presionan también las políticas de la Alcaldía y entonces rápidamente estos barrios son trasladados de estos lugares para lo que se conoce hoy como Villa Santana, Tokio. Tokio es una respuesta de la Administración Municipal a la presencia negra en la ciudad, entonces lo que uno podría a manera de hipótesis subrayar allí, es que para la Administración la manera de resolver la presencia urbana de la gente negra que como te digo, está en el centro ubicada, metida en el centro, y para ellos es feo esos barrios cuando la gente entra y todo el tema asociado al de la inseguridad y todo este cuento, ellos resuelven eso sacando a esta gente de acá y trasladando el problema para otro lado, entonces allí no hay una solución de fondo, allí lo que hay es una jugada política para impedir que estos lugares cercanos al centro se conviertan en lugares peligrosos para los intereses de los mestizos, entonces son reorganizados, y son ubicados en esta parte de acá. ${ }^{27}$

\section{"Uno siempre espera regresar, pero vea"28: proyecto de vida y migración}

Además de las limitaciones que su origen étnico-racial les impone, los jóvenes chocoanos experimentan la discriminación social marcada por las diferencias de clase, no sólo en términos objetivos, sino también en la manera como ellos vivencian en el plano subjetivo su ubicación en los niveles más bajos de la escala social. A la luz de algunos conceptos bourdenaos, en este apartado, nos ocuparemos de las barreras que esta posición representa en las expectativas de los jóvenes hacia su proyecto de vida.

Para Pierre Bourdieu, el objeto de la ciencia social no es el individuo sino el campo social al que pertenece, el cual modela su visión del mundo dependiendo de la posición que éste ocupa dentro del mismo. Los agentes están socialmente constituidos por el campo. Mediante un complejo y prolongado proceso de condicionamiento, éstos adquieren disposiciones específicas o habitus, esto es, aquellos "sistemas perdurables y trasladables de esquemas de

\footnotetext{
${ }^{27}$ Entrevista realizada al Profesor Palacios de la Fundación Universitaria Andina. Pereira, 10.12.2011.

${ }^{28}$ Palabras de Juan, joven chocoano de 19 años, en entrevista realizada en Pereira, el 02.02.2012.
} 
percepción, apreciación y acción que resultan de la institución de lo social en el cuerpo". ${ }^{29}$

A través de estas categorías de percepción y apreciación, los agentes interiorizan los ideales sociales pero también los límites que su propia experiencia social les marca y que condicionan la visión que éstos tienen de su futuro, al reconocer lo que surge en su conciencia bajo la consigna de realidad "no es para gente como nosotros" ${ }^{\prime 30}$. En consecuencia, el horizonte de un futuro de múltiples opciones se reduce para aquellos que, como los afrocolombianos, ocupan posiciones desventajosas en el campo social.

Las estructuras de preferencia de los agentes son, por ende, el producto de una compleja dialéctica entre las oportunidades objetivas y las esperanzas subjetivas, donde estas últimas se hallan poderosamente influidas por los habitus de clase, es decir, la forma interiorizada de la posición de clase y de los condicionamientos que ésta impone a los individuos que se encuentran situados en posiciones sociales similares, generando sistemas de disposiciones homogéneas, y por consiguiente, prácticas semejantes entre ellos. Esta homogeneidad de las disposiciones asociadas a una posición y su adecuación a las exigencias de la misma es, en opinión de Bourdieu, el resultado del ajuste entre las aspiraciones del individuo y las oportunidades objetivas que enfrenta: los agentes desean aquello a lo que están objetivamente destinados ${ }^{31}$. Así, las "afinidades electivas" de los individuos, sus gustos y preferencias, están dictadas por el campo social del que provienen, mismo que ellos tienden a reproducir.

Esta dialéctica entre posiciones y disposiciones se expresa con toda claridad en el ámbito educativo. En sus diferentes trabajos sobre el sistema de enseñanza francés, Bourdieu y Passeron se refirieron a la influencia ejercida por el origen social en las elecciones educativas. Así, las percepciones sobre la viabilidad de la educación superior como un futuro "posible" o "imposible", las representaciones que se tejen en torno a determinadas profesiones como "inalcanzables" por los costos implicados en ellas, así como el desigual acceso a la información sobre las diferentes carreras y sus perspectivas futuras, no son otra cosa que la expresión de la conciencia que los agentes tienen de las posibilidades educativas objetivas de las que disponen, dependiendo de su ubicación en el mapa social ${ }^{32}$.

Las consideraciones de estos autores pueden ser retomadas en bien de un análisis de las expectativas que los jóvenes chocoanos residentes en Pereira abrigan respecto a su proyecto de vida que, si bien se dirigen hacia un deseo

${ }^{29}$ BOURDIEU, Pierre; WACQUANT, Löic. Una invitación a la sociología reflexiva, p. 187.

30 Ibidem, p. 191.

31 BOURDIEU, Pierre, La distinción. Criterios y bases sociales del gusto, p. 109.

32 BOURDIEU, Pierre; PASSERON, Jean-Claude. Los herederos: los estudiantes y la cultura, p. 125; IDEM. La reproducción. Elementos para una teoría del sistema de enseñanza, p. 28. 
de superación para ellos y sus familias a través del empleo o del estudio, se encuentran mediadas por el reconocimiento de las limitaciones objetivas que su condición económica y social les impone. Es así como al indagar por los empleos en los que estos jóvenes buscan desempeñarse, pudimos constatar que, en la mayoría de los casos, las entrevistadas buscan vincularse al servicio doméstico, como cocineras en restaurantes o como vendedoras, mientras que los varones se dirigen hacia el empleo en la construcción. De la misma manera, la vinculación al ejército o la policía es visualizada por unas y otros como una alternativa expedita para la obtención de ingresos, tal y como lo expresa Paula:

Sí, porque es una forma más rápida de salir económicamente, porque ese estudio es de muy largo tiempo, usted escoge una carrera y son cuatro, cinco o seis años, en cambio uno se mete a la policía o al ejército y en uno o dos años ya está trabajando o ganando con lo que va a sobrevivir y la familia. ${ }^{33}$

No obstante este reconocimiento de las limitaciones objetivas que condiciona las expectativas de vida de los sujetos, no podemos perder de vista la misma consideración bourdeana respecto a la capacidad de agencia de estos últimos, cuyas acciones no pueden ser entendidas como una mera reacción mecánica a fuerzas externas que inevitablemente refuerza las estructuras. La capacidad de separarse de dicho orden también cabe dentro de los resultados posibles de dichas acciones. Así, encontramos experiencias como la de Laura, una joven chocoana de 23 años que arribó a la ciudad en el último lustro. Pese a haberse visto en la necesidad de emplearse en el servicio doméstico durante los primeros años de su estadía en Pereira, Laura decide dar un giro a su vida e iniciar sus estudios profesionales. Hoy en día, cuestiona el hecho de que sus amigas no hayan continuado con sus estudios:

L: Porque hay ahí unas, yo tengo unas amigas que llegaron acá también a donde mí, se pusieron a trabajar y no, pues no volvieron a entrar a estudiar, son muchachas jóvenes con capacidades de salir adelante y no, se quedaron en una casa de familia.

E: Bueno, pero y tú, mientras estuviste trabajando en casa de familia, ¿siempre tuviste en mente el proyecto de estudiar?

L: iAh, sí! O sea, porque uno dice, pues yo no sé, hay gente con su cosa, pero yo digo que uno pasa once años pasando trabajo, terminó el bachillerato y pues cómo lo voy a dejar ahí si ha sido bastante trabajo, entonces hay que seguir hasta lograr algo.

E: Claro, poder continuar...

L: O sea, y no, hay mucha gente que no, se estancó ahí y tienen el bachillerato muchos aquí en Pereira y no han podido. ${ }^{34}$

\footnotetext{
33 Entrevista realizada a Paula. Pereira, 11.12.2011.

${ }^{34}$ Entrevista realizada a Laura. Pereira, 27.11.2011.
} 
En cuanto a las elecciones educativas de los jóvenes, podría decirse que éstas obedecen a una anticipación práctica respecto a las posibilidades reales de optar por una u otra profesión. Es así como las aspiraciones de ser cantantes o jugadores de fútbol, pronto ceden el paso a elecciones más realistas como cursar una carrera profesional. Ahora bien, esta última opción se encuentra mediada a su vez por un balance a partir del cual se ponderan distintos factores como la oferta educativa disponible en la ciudad, particularmente en la universidad pública, el costo de las carreras, las facilidades ofrecidas por éstas en términos de un horario que permita distribuir el tiempo entre el estudio y el trabajo y, la oferta de programas de estímulos y becas, de tal suerte que muchas veces los jóvenes terminan estudiando lo que pueden y no lo que quieren. Así lo evidencia el siguiente extracto de la entrevista efectuada a Noelia, una joven de 19 años quien a raíz del asesinato de su padre, tuvo que desplazarse junto con su familia durante el último lustro:

E: ¿Y qué te gustaría estudiar?

$\mathrm{N}$ : Ah estoy viendo, pues a mí siempre me gustó la psicología. No hay plata. Pues vamos a ver qué, como cerraron las convocatorias aquí, el próximo año qué podemos estudiar, sino pues estudiaría filosofía.

E: ¿En la UTP?

N: Sí.

E: ¿Pero en la UTP hay psicología?

$\mathrm{N}$ : No, como es muy cara no hay, entonces toca pues estudiar filosofía y después que uno pueda estudiar una carrera más barata, con eso uno se puede dar lo que uno quiera. Porque uno nunca estudia digamos lo que uno quiere..$^{35}$

Así por ejemplo, entre las elecciones educativas de los chocoanos que estudian en la UTP, se destaca la preferencia de éstos por la Licenciatura en Etnoeducación y Desarrollo Comunitario. La inclinación hacia esta carrera puede explicarse en virtud de su horario nocturno, así como de las monitorías remuneradas que ésta ofrece a sus estudiantes. Es el caso de Laura, quien si bien en un principio no se mostraba muy interesada en esta Licenciatura, terminó inscribiéndose en ella motivada por la posibilidad de trabajar simultáneamente. Así, además de encontrarse cursando esta carrera, Laura se desempeña actualmente como monitora social en la misma universidad. Al preguntarle por las razones que la llevaron a matricularse en dicho programa responde:

L: Porque para poder trabajar y estudiar, porque usted sabe que uno no tiene apoyo de otro, entonces para poder tenerlo todo.

E: ¿Y esa Licenciatura te da la posibilidad de hacer las dos cosas?

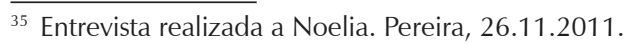


L: Pues, como le dijera, pues a veces me veo muy ocupada que no me queda tiempo de trabajar icierto?, pues sí busca uno el modo, pues sí es muy poquitos los tiempos que me he quedado sin trabajar, y de eso se trata, porque si yo no trabajo cómo voy a estudiar.

$\mathrm{E}$ : ¿Y te gusta el contenido del programa?

L: Sí, ya es que yo me inscribí en él, pues en estas alturas de la vida no puede estar uno allí, 'no me gustó', 'no me gustó', a mi me parece que cuando uno tiene algo claro, si a uno le tocó algo, así no le guste hay que hacerlo y si lo que uno quiere es como sobresalir rápido, entonces no hay que perder tiempo. ${ }^{36}$

Además de esta Licenciatura, los jóvenes entrevistados, particularmente las mujeres, mostraron su predilección por el programa de enfermería. Asimismo, la realización de carreras técnicas en el Servicio Nacional de Aprendizaje -SENA- es visualizada como una alternativa viable de cualificación para el trabajo, o bien como una opción temporal de estudio mientras se da inicio a una carrera profesional.

Es importante mencionar que para aquellos migrantes que han tenido la oportunidad de acceder a una carrera, esta vivencia ha representado una experiencia vital que les ha brindado la posibilidad de ser reconocidos y valorados en el seno de sus familias y comunidades, así como ganar una autonomía de pensamiento antes desconocida. El caso de Milton es elocuente en este sentido. Desde su llegada a Pereira hace cuatro años, Milton, un migrante chocoano de 40 años, ha tenido que hacer frente a toda suerte de vicisitudes para poder materializar su sueño de hacerse profesional: desde resistir largas jornadas repartiendo su tiempo entre el trabajo como obrero en la construcción y la asistencia a las clases en la jornada nocturna, hasta recurrir a las ventas informales en el campus de la universidad para procurarse su sustento y el de su hijo. Pese a las penurias económicas, para Milton su paso por el mundo académico, ha sido una experiencia liberadora:

La academia le da un estado de reconocimiento de uno mismo, de independencia del mundo que a veces lo mantiene atado por el discurso que le monta la sociedad, de la moralidad sesgada, y cuando uno se va desprendiendo de eso, va sintiéndose como más libre y eso le sirve para su estado anímico en gran medida. ${ }^{37}$

Lo cierto es que, en la gran mayoría de los casos, las expectativas de los jóvenes chocoanos frente a su proyecto de vida se encuentran ligadas a la continuación de estudios o a la consecución de trabajo en la ciudad, por lo que el retorno al lugar de origen no es visualizado como la mejor alternativa de futuro. Este hallazgo no es inesperado, pero no por ello menos importante. Asistimos a un proceso de reconfigu-

\footnotetext{
36 Entrevista realizada a Laura. Pereira, 27.11.2011.

37 Entrevista realizada a Milton. Pereira, 01.02.2012.
} 
ración en las expectativas de vida de estos jóvenes, donde la experiencia de vida de los adultos es vista como una experiencia marcada por las privaciones económicas y materiales, y por ende, por un limitado espectro de elecciones disponibles. También el hecho de que una buena parte de los familiares de estos jóvenes se encuentre residiendo en la ciudad, ha modificado el horizonte temporal de su proyecto migratorio, haciendo de este proyecto uno de carácter permanente. Así por ejemplo, Pamela, una joven de 22 años, se expresa de la siguiente manera al ser inquirida sobre la posibilidad de continuar el mismo modus vivendi de sus padres:

P: En realidad no, porque ya la vida de uno (...) uno se plantea algo, una meta y va directamente hacia esa meta, a cumplirla, y uno quiere algo como mejor, algo de lo que ellos no tuvieron y que hoy en día uno tiene la posibilidad de salir adelante, entonces uno prefiere eso, lo mejor, para poder darle a ellos una vida mejor.

E: Y tal vez a ellos les tocó sufrir mucho trabajando en el campo entonces...

P: Claro, desde chiquitos, llegar del colegio, descargar e irse para el trabajo y muchas veces salirse del colegio para coger un marido o para poder salir adelante y renunciar a muchas cosas por los recursos, porque no habían medios para sacar adelante a los hijos, entonces en cambio para mí ahora, lo que a uno le toca vivir ahora, es mucho mejor de lo que les tocaba vivir a ellos, porque ya a uno le dan la posibilidad de darle el estudio, es algo que uno debe de aprovechar al máximo. ${ }^{38}$

En las expectativas de los jóvenes chocoanos frente a su proyecto de vida, interviene la valoración que éstos hacen de las oportunidades objetivas ofrecidas por sus sitios de procedencia en relación con aquellas existentes en la ciudad de Pereira. Aunque los jóvenes echan de menos que las relaciones sociales en sus lugares de origen no estén mediadas por un nivel de monetarización tan acusado como en la ciudad, así como los lazos de solidaridad, la cohesión social, el estrecho vínculo que se construye con el entorno natural, entre otros aspectos, no visualizan oportunidades de superación en ellos. En contraste, la percepción de la ciudad es la de ser el espacio por excelencia para la realización de sus aspiraciones, en virtud de las oportunidades de trabajo, de los niveles de remuneración salarial, de una oferta educativa más amplia y especializada, de las distintas opciones habitacionales, así como del acceso a servicios públicos y a las comodidades que la misma ofrece. En este punto, es pertinente citar en extenso el testimonio de Miguel, un joven migrante de 24 años, quien describe de una manera sin igual el contraste existente entre la forma de vida en el Chocó y en la ciudad:

Quizás desde mi lugar de origen hay cosas que extraño. El encuentro con el agua porque pues nuestro río, algo fundamental porque digamos esa era como la forma de relacionarme mucho. Mucha actividad, mucha tierra, la agricultura como tal, que son cosas muy bonito. La gente que uno siempre

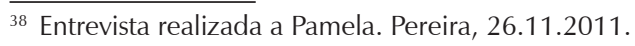


encontrará, o sea en nuestro lugar todos somos familia, el uno es "tío" aunque no sea de sangre, pero siempre se ha tenido como el mayor, siempre será nuestro tío, mientras acá uno empieza a partir diferencias, que siempre está como resumido a cuatro paredes, que es como lo importante usted vivir en su casa y no le importa lo demás, algo que yo veo acá contrastes bastante fuertes, la relación, yo sé que llevo dos, tres años viviendo en un lugar y no sé cómo se llama el vecino, no sé qué hace el vecino. Mientras en nuestra tierra eso es, no sé si es porque sea muy pequeño uno trata de interactuar más con las personas cercanas, pero esos son unos contrastes bastante fuertes lo que uno evidencia. Quizás algo que uno termina de perderse digamos... que en ocasiones dice: 'me encuentro con la noche'. La noche se siente allá como un espacio de paz, de tranquilidad, mientras acá no, nosotros ya... para mí era extraño estudiar en la noche, pero creo que ya después se fue uno adaptando y la noche hace parte como también del día. Entonces uno: 'iah no, son dos de la mañana, tres de la mañana!', acá no tiene importancia ¿cierto?, pero para allá sí, la tranquilidad y el silencio es algo fundamental que uno entiende que es la noche como un lugar de tranquilidad y paz. ${ }^{39}$

Pese a la nostalgia que la remembranza del terruño despierta, los jóvenes chocoanos sienten que su vida ha mejorado a partir de su experiencia en la ciudad, la cual les ha permitido descubrir "otra mirada de las cosas", como lo expresaba una de las entrevistadas. Lo anterior, no obsta para que haya un reconocimiento de las dificultades que entraña la vida urbana, tales como el desempleo, la necesidad de contar con dinero para subsistir y desenvolverse en ella y, la discriminación racial, que no ha sido ajena para ellos.

\section{A manera de conclusión}

Los resultados de esta primera aproximación a los procesos de inserción de los migrantes chocoanos a la ciudad de Pereira coinciden, en lo sustancial, con los encontrados por otros investigadores que se han ocupado de este tema para el caso de Bogotá, Cali o Medellín. De manera general, se pudo establecer que la inserción de la gente chocoana en esta ciudad se ha dado en condiciones de subordinación, lo cual se expresa en distintos ámbitos como el laboral, el espacial y el residencial. Es así como la carencia de recursos económicos, los bajos niveles de cualificación laboral y de escolaridad, así como su condición étnico- racial, restringen las posibilidades de esta población para competir en el mercado de trabajo, situándola, por lo menos inicialmente, en los últimos peldaños de la escala laboral: servicio doméstico, trabajo en la construcción, recolección de café o ventas informales. Esta situación se traduce en sus precarias condiciones de vida en la ciudad, donde el acceso a servicios básicos como la vivienda es una lucha a brazo partido contra las autoridades, los especuladores inmobiliarios y los empresarios privados por hacerse a un espacio en el paisaje urbano.

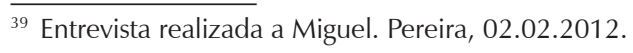


Pero este reducido espectro de opciones disponibles no sólo se traduce en las condiciones materiales de existencia, sino también en la conciencia que los migrantes tienen de las limitaciones objetivas que su condición social, económica y racial les impone. Es así como las expectativas de los jóvenes chocoanos respecto a su proyecto vital, se encuentran mediadas por este reconocimiento, el cual se expresa en el ajuste entre sus aspiraciones laborales y educativas y sus oportunidades objetivas de vida. Así, aunque sus perspectivas están puestas en el deseo de superación mediante el empleo o el estudio, la inclinación por uno y otro trabajo o por ciertas carreras obedece a una anticipación práctica respecto a las posibilidades reales de conseguirlos. Lo anterior, no obsta para que algunos de ellos le hayan dado un giro a su vida, logrando con ello, torcer el camino trazado por estas limitaciones.

Así, el análisis de lo que significa para un joven afrocolombiano y pobre insertarse en el contexto urbano nos permite descubrir cómo en su experiencia migratoria se entrelazan de manera simultánea los distintos tipos de desigualdad social, marcados tanto por su pertenencia étnico-racial, como por su situación de clase. En términos teórico-metodológicos, la forma particular como se expresa el fenómeno de la desigualdad para los afrocolombianos advierte la creciente necesidad de asumir perspectivas de análisis interseccionales, desde las cuales se aborden las complejas interacciones existentes no sólo entre las categorías étnico-raciales y de clase social, sino también entre el sexo-género, la orientación sexual y el ciclo de vida.

Ahora bien, el espacio de realización de las aspiraciones de los jóvenes no es otro que el de la ciudad, la cual les resulta más atractiva que sus comunidades de origen, en razón a distintos factores como la mayor infraestructura educativa, el mercado laboral más abierto, las alternativas habitacionales, el dinamismo de las actividades culturales y la oferta de servicios básicos y de comodidades de las que carecen en las localidades chocoanas. En efecto, la creciente exposición de los jóvenes a nuevos referentes de orientación cultural como los procesos de escolarización, el acceso a trabajos mejor remunerados, los medios de comunicación, los recursos tecnológicos y el contacto con otros grupos sociales en la ciudad, han representado una reconfiguración de sus aspiraciones de vida, donde las expectativas de retorno a su terruño, les son cada vez más ajenas.

\section{Bibliografía}

AGIER, Michel; BARBARY, Oliver; HOFFMANN, Odile; QUINTÍN, Pedro; RAMÍREZ, Héctor; URREA, Fernando. Espacios regionales, movilidad y urbanización, dinámicas culturales e identidades de las poblaciones afrocolombianas del Pacífico sur y Cali. Una perspectiva integrada. Informe Síntesis. Proyecto Cidse-IRDColciencias, Documento de Trabajo No. 52. Cali: Cidse, Univalle, 2000.

BARBARY, Olivier. Observar los hogares afrocolombianos en Cali, problemas teóricos 
y metodológicos ilustrados. In BARBARY, Olivier; URREA, Fernando (coords.). Afrocolombianos en el área metropolitana de Cali: estudios sociodemográficos. Documento de Trabajo No. 38. Cali: Cidse, Univalle, 1999, p. 5-30.

BARBARY, Olivier; URREA, Fernando. Introducción. In Gente negra en Colombia. Dinámicas sociopolíticas en Cali y el Pacífico. Medellín: Ed. Lealon, 2004, p. 17-65. BOURDIEU, Pierre. La distinción. Criterios y bases sociales del gusto. Madrid: Taurus, 1999.

BOURDIEU, Pierre; PASSERON, Jean- Claude. Los herederos: los estudiantes y la cultura. Buenos Aires: Siglo XXI Editores, 2009 [1964].

. La reproducción. Elementos para una teoría del sistema de enseñanza. México: Distribuciones Fontamara, 1996.

BOURDIEU, Pierre; WACQUANT, Löic. Una invitación a la sociología reflexiva. Buenos Aires: Siglo XXI Editores, 2005.

CALDERÓN, Juan. Propuesta de constitución legal y administrativa para un grupo de mujeres en condición de vulnerabilidad económica, habitantes de los Barrios Caracol La Curva y El Bosque, aledaños al "Salado de Consotá, Pereira. Tesis de licenciatura. Pereira: Universidad Tecnológica de Pereira, 2011.

DE LOS MILAGROS, Camilo. Pereira: etimología de las invasiones. Foro de acceso libre en la página Rebelión, 2012. Disponible en: < http://www.rebelion.org/ noticia.php?id=146538>. Consultado: 05.04.2012.

HENAO, Marisol. Una aproximación al desarrollo de habilidades comunicativas en la comunidad desplazada "del Plumón Alto" a través de la comunicación asertiva. Tesis de licenciatura. Pereira: Universidad Tecnológica de Pereira, 2007.

LÓPEZ, Santiago; SOTO, Stefany. Gestión ambiental urbana en programas de vivienda popular; estudio de caso "Ciudadela Tokio", Pereira, Risaralda. Tesis de licenciatura. Pereira: Universidad Tecnológica de Pereira, 2011.

MOSQUERA, Claudia. Estrategias de inserción de la población negra en Santafe de Bogotá. Acá antes no se veían negros. Santa Fe de Bogotá: Observatorio de Cultura Urbana, Instituto Distrital de Cultura y Turismo, 1998.

POSSO, Jeanny. La inserción laboral de las mujeres inmigrantes negras en el servicio doméstico de la ciudad de Cali. Cali: Universidad del Valle, 2008.

RIVERA, Alberto. La reubicación es un hecho. Periódico La Tarde. Pereira: 24.11.2006. TOBÓN, Alfredo. Barrio Cuba, una ciudad dentro de otra. El diario del Otún. Pereira: 03.07.2011.

TROYA, Marcela. Entre el recuerdo y el presente del Aeropuerto Internacional Matecaña. Foro de acceso libre en la página Pereira iTu Ciudad!, 2011. Disponible en: <http://www.pereiratuciudad.com/es/ieventos/ver/284/entre_el_recuerdo_y_ el_presente_del_aeropuerto_internacional_matecana/>. Consultado: 05.04.2012.

URREA, Fernando; VIÁFARA, Carlos; CORREA, Juan. Desigualdadessociodemográficas y socioeconómicas, mercado laboral y discriminación étnico-racial en Colombia: análisis estadístico como sustento de Acciones Afirmativas a favor de la población afrocolombiana. In MOSQUERA, Claudia; LEÓN, Ruby (eds.). Acciones afirmativas 
y ciudadanía diferenciada étnico-racial negra, afrocolombiana, palenquera y raizal. Entre Bicentenarios de las Independencias y Constitución de 1991. Bogotá: Universidad Nacional de Colombia, 2009, p. 153-346.

WADE, Peter. Gente negra, nación mestiza: dinámicas de las identidades raciales en Colombia. Bogotá: Ediciones Uniandes, 1997.

\section{Abstract}

"The sun does not always shine for everyone": insertion strategies of young Afro-Colombians into the city of Pereira

The accelerated urbanization process of the Afro-Colombian population can be explained from the growing migratory flow from the Pacific region toward the cities; among which, Pereira has acquired relative importance in recent years as a pole of attraction for this population. Nevertheless, the migratory phenomenon toward this city, as well as integration processes of migrants onto the receptor society have received little attention from social analysts. In this sense, this paper seeks to contribute to clarifying the urban insertion strategies deployed by Afro-Colombian migrants, highlighting the specific experience of young men and women coming from the department of Chocó, because they constitute one of the population groups that are most strongly impacted by said phenomenon. In light of this case study, we intend to show that, by virtue of their ethnic-racial heritage, regional origin, and social class, these young people encounter adverse conditions in their integration process onto urban life

Keywords: migratory process, urban insertion strategies, young AfroColombians, Pereira, Chocó.

Recebido para publicación en 27/02/2013.

Aceptado para publicación en 29/07/2014. Received for publication in February, 27 $7^{\text {th }}, 2013$.

Accepted for publication in July, 29 2014. 